\title{
Distance et proximité entre plusieurs discours dans l'argot journalistique de la revue Variety
}

François Ropert

\section{(2) OpenEdition}

1 Journals

Édition électronique

URL : http://journals.openedition.org/asp/222

DOI : $10.4000 /$ asp. 222

ISBN : 978-2-8218-0408-1

ISSN : 2108-6354

\section{Éditeur}

Groupe d'étude et de recherche en anglais de spécialité

Édition imprimée

Date de publication : 1 mars 2009

Pagination : 23-43

ISSN : 1246-8185

\section{Référence électronique}

François Ropert, « Distance et proximité entre plusieurs discours dans l'argot journalistique de la revue Variety », ASp [En ligne], 55 | 2009, mis en ligne le 01 mars 2012, consulté le 04 novembre 2020 URL : http://journals.openedition.org/asp/222 ; DOI : https://doi.org/10.4000/asp.222

Ce document a été généré automatiquement le 4 novembre 2020.

Tous droits réservés 


\title{
Distance et proximité entre plusieurs discours dans l'argot journalistique de la revue Variety
}

\author{
François Ropert
}

1 La revue Variety se présente comme une publication éclectique consacrée aux nouveautés dans le domaine du divertissement. Au-delà du pouvoir évocateur de son nom, sa souplesse éditoriale est attestée dès ses origines comme dans ses abondantes archives ainsi que dans ses éditions hebdomadaires et quotidiennes les plus récentes. Revue corporatiste et spécialisée, Variety œuvre depuis sa création à publier toute information utile aux professionnels du spectacle et notamment du cinéma. Revue grand public, elle veille également à couvrir l'actualité qui présente le plus d'attrait pour le lecteur de base, dont les intérêts n'ont cessé de se diversifier. En première analyse, ces différents paramètres sont révélateurs de la distance qui sépare plusieurs lectorats dans le temps et dans l'espace socioprofessionnel et culturel du monde du spectacle. Cependant, l'étude des évolutions de Variety tout au long de son histoire démontre que l'éclectisme de la revue favorise un positionnement éditorial équilibré et fédérateur entre des intérêts économiques et artistiques divergents. Ceci se vérifie ponctuellement en période de grève dans l'industrie du cinéma; la revue maintient aussi ce type de positionnement face à l'opposition structurelle entre les majors et le secteur indépendant, ou encore à l'égard de compromis commerciaux et artistiques, dont résulte par exemple la notion de genre cinématographique. À partir des enjeux phonologiques et sémantiques d'une manchette historique, à laquelle le slanguage doit la célébrité ${ }^{1}$, il apparaît que cet argot constitue un ressort essentiel à la souplesse éditoriale de Variety. Il établit un lien sociolinguistique entre les nombreux lecteurs de la revue. Il parvient également à capter différents types de discours dans un faisceau qui balaye plusieurs domaines d'intérêts et de compétences, dont il peut à la suite enrichir la terminologie.

2 Après avoir situé la fonction éditoriale du slanguage dans l'histoire de la revue et le contexte économique et artistique de l'industrie du divertissement, notre étude se 
resserre autour d'une typologie de règles lexicogéniques applicables au slanguage. Il en ressort que les créations lexicales de cet argot promeuvent une forme d'exclusivité journalistique relayée par le discours écrit, exclusivité qu'elles contribuent cependant à mettre en scène et à centrer virtuellement autour de la voix et du dialogue. Le slanguage présente donc une forte dimension phatique et visuelle. Certaines de ses créations lexicales donnent également lieu à la formation d'une terminologie de type néologique. Qu'elle soit de forme ou de sens, cette néologie, en évolution constante, détourne tout phénomène de déterminologisation et stimule une métaphorisation constante du discours. En dernière analyse, cette métaphorisation paraît instaurer une distance critique à l'égard de discours formatés sur des pratiques professionnelles et commerciales, ou à l'égard d'une tendance au métadiscours dans le discours critique lui-même. Cette mise à distance doit encore être nuancée. Elle reste le fait d'une néologie en clin d'œil, à mi-chemin entre humour et ironie. Elle participe d'un effet de brouillage discursif en établissant une proximité complice entre la revue et ses lecteurs dans toute leur diversité.

\section{L'éclectisme d'une revue corporatiste, spécialisée et grand public}

3 La ligne éditoriale de la revue s'est définie dans le but de fédérer un très large lectorat. Revue corporatiste et spécialisée, mais également grand public, Variety se caractérise par son éclectisme. L'objet de cette première partie est de présenter le projet fondateur de la revue et de suivre ses évolutions. Celles-ci ont été conditionnées par les mutations de l'industrie du divertissement et par l'évolution des goûts du public depuis plus d'un siècle, dans un contexte de concurrence propre au secteur de la presse généraliste et spécialisée. Revue plus que centenaire, Variety offre un matériau riche et abondant pour saisir ces différents enjeux dans une perspective historique qui couvre l'actualité du divertissement, depuis l'essor du cinéma jusqu'au développement des nouvelles technologies de l'information'2.

\subsection{Le projet fondateur d'une revue corporatiste et grand public}

Lorsque Sime Silverman (1873-1933) lance le premier numéro de Variety à New York, le 16 décembre 1905, il peut compter sur le soutien de la scène new-yorkaise. Variety se présente comme une revue corporatiste en étroite proximité avec le monde professionnel du spectacle, mais dont l'objectif est aussi de fournir un relais médiatique novateur entre les spectacles de Broadway, du off Broadway - et même du off off Broadway - et les non-professionnels. En effet, la presse généraliste tenant le grand public trop à distance de l'extrême variété des productions à l'affiche (Stoddart 1941:122-173), Variety couvre donc à ses débuts toute l'actualité théâtrale, en s'adressant à un lectorat varié de professionnels et de spectateurs avertis ou non.

\subsection{Une revue éclectique}

5 L'éclectisme de Variety est ainsi un trait distinctif de la revue dès sa création. Elle ouvre aussi très rapidement ses colonnes à des spectacles plus populaires que le théâtre : les cirques, les fêtes foraines et les cabarets. La ligne éditoriale de Variety rapproche, d'une 
part, ces différents styles de spectacles tenus à distance les uns des autres, souvent par le mépris ou l'ignorance dans le reste de la presse. D'autre part, au-delà de toute limite corporatiste, Variety a aussi pour vocation d'informer le grand public des nouveautés parmi les plus petits spectacles et les moins onéreux. Cet éclectisme s'est affirmé tout au long de l'histoire de la revue, notamment à partir de l'apparition d'une nouvelle forme de divertissement populaire : le cinéma.

\subsection{Une revue spécialisée}

Variety a prêté une grande attention à l'essor du cinéma, un nouveau genre de divertissement ouvert à un très large public, qu'il soit citadin ou non, cinéphile ou moins averti (Scott 2005 : 11-35). La revue s'est très tôt positionnée pour devenir une publication incontournable dans la presse spécialisée du secteur, au fur et à mesure que le cinéma a consolidé son double statut de secteur économique majeur et d'activité artistique à part entière. The Hollywood Reporter a été le premier concurrent direct de Variety dans le secteur. Cette revue rivale a été créée le 3 septembre 1930, laissant à Variety une certaine avance pour établir sa notoriété. Dès 1933, Sime Silvermana néanmoins répondu à cette concurrence en délocalisant une partie de ses bureaux à Los Angeles $^{3}$. Cette délocalisation partielle appuyait les nouveaux choix éditoriaux de Variety. Grâce à elle, les journalistes de la revue qui étaient installés à Los Angeles pouvaient couvrir l'actualité cinématographique aussi rapidement que les équipes californiennes du Hollywood Reporter, soit quasiment en temps réel.

\subsection{Concurrence et rythme de parution}

7 La concurrence entre Variety et The Hollywood Reporter a dès lors pris la forme d'une course à l'exclusivité. Sime Silverman a mis en œuvre tous les moyens possibles pour rendre sa revue toujours plus réactive à l'actualité et pour obtenir les derniers scoops. L'implantation californienne de Variety a donc, avant tout, été conditionnée par les impératifs d'un rythme de parution adaptée à celui de l'actualité des derniers films à l'affiche. Elle a permis la publication d'une édition quotidienne produite à Los Angeles, à l'instar du Hollywood Reporter et pour ainsi mieux rivaliser avec ce nouveau concurrent. L'édition californienne de Variety paraît sous le titre de Daily Variety, afin que les lecteurs la distinguent clairement de l'édition hebdomadaire new-yorkaise qui conserve le titre original Variety. Celle-ci a, depuis cette époque, traité en priorité de l'actualité théâtrale, plus spontanément associée à la scène new-yorkaise et à la côte est des États-Unis. Quant à l'actualité cinématographique, elle y est couverte globalement et sous la forme de recensions régulières des productions les plus marquantes. La marque distinctive de Daily Variety est, en revanche, de fournir des critiques immédiatement dès les premières, c'est-à-dire en moyenne deux semaines plus tôt que l'hebdomadaire publié à New York.

\subsection{Une ligne éditoriale souple}

8 L'éclectisme et la spécialisation de Variety témoignent à l'évidence d'une ligne éditoriale souple. Cette souplesse nécessite une grande réactivité de la part de la revue face aux mutations de l'industrie du divertissement, face à la concurrence dans le secteur de la presse, et face aux évolutions des intérêts du public et donc de son 
lectorat. Cette réactivité a caractérisé les évolutions éditoriales de la revue depuis plus d'un siècle. Aux deux éditions historiques se sont régulièrement ajoutées de nouvelles éditions, qu'elles soient hebdomadaires ou quotidiennes ${ }^{4}$. Elles ont été rendues nécessaires par la diversification de l'industrie du divertissement, depuis l'apparition de la télévision jusqu'à celle des jeux vidéo et des nouvelles technologies (Scott 2005 : 35-61). Tout en maintenant une proximité étroite avec le cinéma, la ligne éditoriale de Variety n'a donc eu de cesse de se diversifier sur le fond et dans la forme. Une édition en ligne est créée en $1998^{5}$. Son lancement enregistre un retard d'à peine trois ans sur l'édition en ligne du Hollywood Reporter, qui a été inaugurée fin $1995^{6}$.

\section{Le positionnement éditorial de la revue}

Revue corporatiste, spécialisée et grand public, Variety se doit de refléter une grande diversité de points de vue et d'intérêts. Son positionnement éditorial en cas de grève d'une part présente un intérêt particulier pour juger de sa souplesse à l'égard des corporatismes. D'autre part, en dehors des situations de crise, il est intéressant d'examiner comment la revue se positionne face aux intérêts économiques des majors et, conjointement, la façon dont elle évalue l'intérêt artistique du cinéma de genre hollywoodien. Ce positionnement dépend en grande partie du rapport de distance et de proximité que la revue établit en parallèle avec le secteur indépendant, situé en marge du marché et proposant une alternative esthétique aux productions commerciales. De ce rapport dépend plus largement l'éclectisme de la couverture de Variety, destinée à refléter l'activité du secteur cinématographique dans son ensemble et à adhérer aux goûts du public cinéphile dans toute sa diversité. L'étude d'une manchette peut servir de test pour vérifier l'imbrication de ces paramètres au niveau du discours.

\subsection{Des intérêts composites}

$\mathrm{Au}$ cœur des débats qui animent Hollywood en période de grève se trouve une problématique récurrente dans l'histoire de l'industrie du divertissement. Elle rapproche et oppose les intérêts composites d'une industrie tentaculaire, aujourd'hui mondialisée grâce aux nouvelles technologies, et les intérêts corporatistes et sectoriels de chacune de ses composantes (Scott 2005 : 138-159). Cette divergence d'intérêts se manifeste autour d'un conflit, ouvert ou latent, qui oppose les impératifs économiques d'une production de masse à des revendications d'ordre artistique. La dernière grève des scénaristes, qui a secoué Hollywood en 2007-08, est un exemple emblématique de la manière dont ce conflit peut se manifester dans toute son amplitude. La couverture de ces récents événements par Variety peut donc, en premier lieu, servir de test à la souplesse de la ligne éditoriale de la revue.

\subsection{Un triple équilibre}

11 Face à des intérêts industriels divergents, exacerbés en cas de grève, Variety témoigne d'une couverture contrastée. Comme lors du mouvement de 1988, Variety a amplement relayé les évolutions du conflit de 2007-087. Variety s'est fait l'écho fidèle des deux groupes professionnels en conflit. Elle a relayé les revendications de la Writers Guild of America (WGA), qui demandait l'obtention d'un pourcentage plus élevé de droits 
d'auteurs sur la vente de produits dérivés. Ces revendications nécessitaient la remise à plat des accords précédemment conclus entre ce syndicat des scénaristes et Alliance of Motion Picture and Television Producers (AMPTP), le puissant syndicat des producteurs, auquel Variety a aussi régulièrement ouvert ses colonnes. Variety a également couvert les réactions des spectateurs confrontés à l'absence de tout nouveau programme télévisé. Sa couverture du conflit a ainsi reflété un triple équilibre qui correspondait parfaitement aux trois dimensions du mouvement, généralement présenté comme un bras de fer entre scénaristes et producteurs. Or, le public était bien, lui aussi, au cœur du conflit. Ses réactions étaient utilisées, tour à tour, par le syndicat des scénaristes et par celui des producteurs pour faire pression sur le camp adverse. L'enjeu corporatiste du conflit recouvrait en effet un enjeu commercial lié à l'intérêt des spectateurs pour les rediffusions, et donc au fléchissement des taux d'audience en l'absence de tout nouveau programme. Variety a donc su, pendant toute cette période, maintenir une forme d'équidistance éditoriale entre les intérêts divergents de ces trois parties. Elle a démontré, en d'autres termes, la souplesse de sa ligne éditoriale, et elle n'a pas failli au principe d'éclectisme qui la caractérise depuis sa création.

\subsection{Majors et secteur indépendant}

12 En dehors de ces situations de crise, la couverture que la revue consacre respectivement aux productions des majors et à celles du secteur indépendant peut, en second lieu, servir de test, dans la durée cette fois, à son positionnement face aux intérêts économiques et artistiques souvent divergents de ces deux secteurs. Sur ce point, les colonnes de Variety reflètent tout à la fois une grande proximité éditoriale avec les dernières stratégies commerciales de l'industrie hollywoodienne, par l'intérêt qu'elle leur porte, et une distance critique par rapport aux moyens que cette industrie utilise pour mettre en œuvre ces stratégies, notamment leur traduction artistique à l'écran. En raison même de la proximité éditoriale que Variety chercheà entretenir à l'égard des dernières créations, la revue doit en effet garder une distance critique à l'égard des productions hollywoodiennes les plus formatées et donc les moins créatives. Le secteur indépendant offre alors à la revue une grande diversité de productions, dont elle couvre régulièrement l'actualité afin de promouvoir la rupture esthétique que ce secteur représente face à Hollywood (Amiel \& Couté 2003 : 31-34).

\section{4. Équidistance et équilibrage éditoriaux}

Cette configuration de l'industrie cinématographique, qui le plus souvent oppose les impératifs économiques d'une production de masse aux revendications artistiques d'un secteur situé en marge des forces du marché, a déjà été observée en cas de grèves. Elle est a priori un obstacle à l'équidistance éditoriale que la revue cherche à maintenir entre plusieurs parties. Or, la revue surmonte cet obstacle par un équilibrage éditorial constant. Le triple équilibre opéré lors de grèves n'est ainsi qu'un aspect d'un équilibrage éditorial qui se vérifie dans la durée et à de multiples niveaux. Dans le cas présent du positionnement de Variety à l'égard des majors et du secteur indépendant, un équilibrage qualitatif est repérable entre les colonnes consacrées aux productions à gros budget et celles, plus épisodiques mais de ce fait plus remarquées, que Variety consacre aux productions indépendantes américaines et étrangères. Variety a contribué, par exemple, à promouvoir la carrière de Steven Soderbergh jusqu'à la 
Palme d'Or au Festival de Cannes,depuis le festival du film de Sundance, quelques mois plus tôt, lorsqu'un journaliste de la revue s'est enthousiasmé pour le film Sex, Lies and Videotape (1989) ${ }^{8}$. Variety a également fait bon accueil au film Reservoir Dogs (1992) pour lequel Quentin Tarantino a été sélectionné à Sundance, avant qu'il ne remporte lui aussi une Palme d'Or à Cannes pour Pulp Fiction (1994) ${ }^{9}$.

\subsection{Le traitement du cinéma de genre}

Un autre aspect de cet équilibrage qualitatif, garant de l'équidistance éditoriale de Variety entre majors et secteur indépendant, se vérifie au niveau du positionnement de la revue à l'égard du cinéma de genre. Celui-ci cristallise à lui seul les tensions d'un compromis entre production de masse et une stylisation esthétique propre aux lois du genre, définies par ce qui fait recette au box-office : une forme de poétique donc, mais à vocation industrielle et commerciale (Amiel \& Couté 2003: 15-19). La souplesse éditoriale de Variety permet à la revue d'adhérer à ce compromis et même de le conforter par le grand nombre de colonnes qu'elle consacre au cinéma de genre. Cependant, si l'actualité de ce type de films est amplement couverte sur le plan quantitatif, la revue publie régulièrement des articles plus incisifs qui posent ouvertement les limites du compromis sur lequel repose la notion même de genre cinématographique. Le poids éditorial de ces articles est d'autant plus grand qu'ils sont moins représentatifs de la revue et donc plus remarqués. C'est ainsi que peut s'opérer un équilibrage également qualitatif. Ce type d'équilibrage permet à Variety de toucher un lectorat dont l'intérêt pour le cinéma dépasse les limites des productions hollywoodiennes. Pour illustrer le positionnement de Variety entre ces intérêts et ces revendications apparemment inconciliables, sa manchette la plus célèbre, Sticks Nix Hick Pix ${ }^{10}$, peut servir d'exemple. Elle porte sur le lancement d'un genre nouveau pour les films hollywoodiens de série B : le genre rural et provincial.

\section{6. « Sticks Nix Hick Pix »}

Ce nouveau genre cinématographique dépeint la vie dans les campagnes. Son cœur de cible est l'Amérique profonde. Sur le plan phonologique, les quatre monosyllabes de cette accroche constituent, par paronomase, un raccourci discursif jubilatoire, en saisissant par un trait d'humour les limites génériques de ce type de production. Ces quatre monosyllabes ont suscité de nombreux commentaires lors de leur parution à la une le 17 juillet 1935. Cette seule manchette a suffi pour établir la célébrité du headlinese, une sous-catégorie du slanguage quand il est utilisé dans les gros titres. Aujourd'hui encore, la formule fait l'objet de fréquentes citations et de nombreux commentaires dans les ouvrages consacrés aux genres cinématographiques. Rick Altman, par exemple, considère la parution de cette manchette comme une date marquante dans l'histoire du cinéma de genre, telle qu'il est perçu par la presse, les critiques et le public. Il y voit plus encore un raccourci discursif inégalé, souvent repris dans le discours universitaire, en raison de sa capacité à fixer, dans une seule formule, les limites esthétiques et commerciales du cinéma de genre hollywoodien dans son ensemble (Altman 1999 : 22-41). 


\section{La souplesse morphologique et sémantique du slanguage}

16 À partir de ces remarques sur l'histoire de Variety, il s'agit maintenant d'évaluer dans quelle mesure le slanguage participe, au niveau du discours, à la souplesse éditoriale et à l'éclectisme de la revue. Nous distinguons, d'une part, les procédés morphosémantiques qui confèrent aux créations lexicales du slanguage leur longévité discursive, dont atteste aujourd'hui encore une manchette comme Sticks Nix Hick Pix. Cette longévité ne doit pas cependant être envisagée comme un obstacle à la capacité de renouvellement propre à ces créations. Ces dernières ont évolué au rythme des transformations de l'industrie du cinéma et des évolutions du discours spécialisé propre à ce secteur. Il s'agit, d'autre part, d'évaluer la capacité du slanguage à migrer d'un registre de langue à l'autre, d'un type de discours à l'autre, et d'une communauté sociolinguistique à l'autre, comme l'atteste, par exemple, leur intégration dans le discours critique et universitaire.

\subsection{Premier aperçu}

Le mot slanguage, créé par Variety à la suite du succès rencontré par la manchette Sticks Nix Hick Pix, résume à lui seul tous ces enjeux. Il donne à comprendre et à imaginer la plasticité d'une langue argotique, qui procède par raccourcis, mélanges et compositions ${ }^{11}$. Ce premier aperçu est développé dans la suite de notre étude au travers d'une typologie des règles lexicogéniques (Tournier $1987: 16-19)$ caractéristiques de cet argot: lexicalisation de logos, de formes graphiques, d'onomatopées, d'initiales et autres abréviations ${ }^{12}$. Cette typologie est assortie d'une analyse du discours et des pratiques langagières d'une communauté socioprofessionnelle et socioculturelle disparate, mais que le slanguage tend à fédérer.

\subsection{La lexicalisation de logos et de formes graphiques}

18 Le slanguage peut superposer message visuel, souvent d'origine industrielle et publicitaire, et discours. De nombreux logos et formes graphiques sont lexicalisés et intégrés dans un titre ou dans le corps d'un article : the Lion, Lion ou Leo en référence à Leo the Lion, le logo légendaire de la Metro-Goldwyn-Mayer ; the Peacock en référence au paon de la NBC. Toutes ces créations lexicales témoignent d'une familiarité de ton qui se démarque du respect que suscitent généralement ces institutions. Elles connotent une forme de proximité entre ces institutions, la revue et les lecteurs. Il en résulte un triple rapprochement qui reflète une communauté d'intérêts, tout en estompant les rapports inégaux entre les éléments qui la constituent. Ce triple rapprochement confirme la valeur strictement argotique de ces créations, puisque l'argot se définit notamment comme

un dialecte social réduit au lexique, de caractère parasite (dans la mesure où il ne fait que doubler, avec des valeurs affectives différentes, un vocabulaire existant), employé dans une couche déterminée de la société qui se veut en opposition avec les autres. (Dubois et al. $2001:$ 48)

19 Peut être ici mentionnée une autre création lexicale qui relève aussi de la constitution d'un bestiaire investi d'une forte dimension affective : Mouse, the Mouse ou Mouse House, 
pour désigner les studios Disney. À la différence des exemples précédents, la lexicalisation procède dans ce cas précis de la forme graphique associée à un personnage familier, petit par la taille mais assez emblématique pour incarner toute la puissance économique d'un conglomérat. Cette familiarité de ton est donc à l'origine de nombreuses créations du slanguage. Elle participe d'un registre de langue familier. Elle donne lieu de surcroît à des mots inédits qui justifient, à plus d'un titre, leur appellation de mots argotiques du fait de leur caractère non normatif, destiné à établir une distance avec la langue courante dans le cadre d'un rapprochement d'ordre sociolinguistique autour de leur emploi.

\subsection{La lexicalisation d'onomatopées et d'initiales}

20 L'effet de paronomase issu de la diphtongaison dans Mouse House est loin d'être un cas isolé dans le slanguage. Celui-ci tend très fréquemment à jouer sur les sonorités, souvent pour réduire l'écart graphie / phonie. C'est le cas pour la lexicalisation d'onomatopées ou de sons qui pourraient, par exemple, exprimer l'enthousiasme. Whammo s'utilise comme adjectif, ou adverbe, pour qualifier un film rencontrant un très vif succès auprès du public: Men in Black is doing whammo overseas. Ce jeu sur les sonorités préside également à la lexicalisation d'abréviations sous forme d'initiales dont la graphie est déformée : a new ayem show pour a new a.m. show, to emcee the Oscars pour to m.c. the Oscars. La distance se creuse alors entre deux signifiants, l'un connu et l'autre inédit, mais toujours dans le but de réduire l'écart graphie/phonie. Ayem évoque graphiquement le bâillement d'un téléspectateur encore endormi devant une émission matinale. Quant au redoublement du $e$ dans emcee, il marque l'allongement du son [i : ] pour rendre au plus près toute la solennité et l'emphase du rôle de maître de cérémonie lors de la soirée des Oscars. L'effet est insolite; il semble se prêter à la réalisation graphique d'un son ou d'un style d'élocution. La lexicalisation de ces initiales peut alors concourir à une mise en scène du quotidien du lecteur, tout comme des fastes d'Hollywood dont semble nous parvenir un écho prolongé sur le papier. L'interprétation que le lecteur en fait au niveau du discours pourra être d'ordre humoristique, voire ironique, à l'égard d'un format télévisuel ou d'un grand événement commercial, artistique et médiatique.

\subsection{Siglaison sous forme de rébus}

21 À l'opposé de cette lexicalisation presque explicative, le slanguage peut recourir à la lexicalisation d'initiales dont l'effet est d'estomper le signifiant. L'écart signifiant / signifié semble du coup en être accru. D'une part, l'assemblage graphique de ces initiales est sans précédent. D'autre part, il réunit par siglaison les initiales d'au moins trois éléments constituant une locution, et la forme siglée résultante se prête très difficilement à une réalisation de type phonologique, car le nombre de consonnes compromet toute prononciation syllabique. Citons par exemple : Her o.t.t. performance marred the otherwise riveting film. 0.t.t. est ici la forme siglée de la locution over the top. Un effet similaire est produit dans la phrase suivante: The outlook for long term B.O. is n.s.g. L'effet insolite vient dans ce cas de la siglaison n.s.g., telle qu'elle est renforcée par contraste avec la forme siglée très connue B.O. pour box-office. Le lecteur de Variety comprend not so good. L'accroissement de l'écart signifiant / signifié est ici destiné à rapprocher les lecteurs qui connaissent le code pour le réduire. Notons que ces formes 
siglées inédites sont le plus souvent adjectivées, ce qui contribue à un rapprochement syntaxique étroit avec un substantif auquel la locution d'origine ne serait pas nécessairement associée. Au niveau sémantique, elles véhiculent donc un jugement de valeur que seuls les initiés au slanguage, et par extension au monde du cinéma, semblent être capables de comprendre et de vraiment apprécier. Seraient tenus à distance tous ceux qui ne maîtriseraient pas le code pour les interpréter. Toutefois, ces formes siglées prennent rapidement à la lecture la forme de rébus dont l'élucidation repose avant tout sur l'inférence. Leur encodage présente un intérêt essentiellement ludique. Plus que d'exclure une partie des lecteurs, il a pour but principal de stimuler leur imagination.

\subsection{Troncations par apocopes}

22 Ces formes siglées sont à rapprocher d'un autre type d'abréviations qui relève quant à lui de la troncation. L'écart signifiant / signifié est moins insolite, puisque l'effet d'estompe provoqué par ces troncations n'est que transitoire avant que la graphie du mot usuel ne réapparaisse dans son intégralité.

Tableau 1. Troncations par apocopes

\begin{tabular}{|l|l|}
\hline Slanguage & Correspondance \\
\hline \hline auds & audience \\
\hline biopic & biographical picture \\
\hline doc & documentary \\
\hline indie & independent \\
\hline perf & performance \\
\hline pic & picture \\
\hline pics / pix & pictures \\
\hline toon & cartoon \\
\hline
\end{tabular}

La première remarque que suggèrent ces exemples (cf. tableau 1) est que ces troncations sont d'origine phonétique et procèdent donc par apocope : l'élision se fait sur les dernières syllabes non accentuées du mot sauf exception toon, et ce pour des raisons évidentes d'homophonies ambiguës (car). La seconde est que les bisyllabiques sont moins fréquemment apocopés que les autres polysyllabiques. Enfin, ces troncations par apocopes participent d'un discours oral et confirment le caractère argotique du slanguage.

\subsection{Créations lexicales et exclusivité journalistique}

L'emploi de la plupart des créations lexicales rencontrées précédemment reste exclusif de Variety. Il relève de l'hapax et constitue finalement une marque de fabrique par le 
ton inimitable qu'elles confèrent à la revue. Même si son origine n'est pas mentionnée, quelques lignes suffisent pour reconnaître un article de Variety. Le slanguage s'élabore donc à partir de créations lexicales dont l'exclusivité redouble, au niveau du discours, l'exclusivité journalistique dont un article est porteur. Cependant, cette exclusivité ne vaut, dans les deux cas, que si elle est partagée par le plus grand nombre de lecteurs. Le slanguage contribue ainsi à la synthèse de la confidentialité qui conditionne la création d'un scoop et de la révélation publique qui en confirme le succès médiatique. Il exclut les lecteurs qui n'en connaissent pas les codes, mais sans que ceux-ci soient totalement hermétiques pour des raisons commerciales évidentes. Au contraire, toutes les abréviations du slanguage tirent en l'occurrence leur intérêt éditorial de la production d'un discours simplifié, au plus proche du discours oral. Or, cette proximité, caractéristique d'un registre familier, favorise l'échange et permet qu'un dialogue virtuel se noue entre la revue et ses lecteurs, autour de références investies d'une valeur affective.

\subsection{Mise en scène du discours et vococentrisme}

Le slanguage est, en d'autres termes, un vecteur pour que la voix du critique passe par le discours écrit et que s'opère cet échange reposant sur l'anticipation des réactions du lecteur à chacune de ces références. Le ton de la revue est donné par le grain de cette voix virtuelle que le slanguage parvient à rendre présente, par réduction de l'écart graphie / phonie, et réussit à mettre en scène dans un réseau de références affectives, par jeu sur l'écart signifiant / signifié. Ce double processus donne au discours une forte dimension phatique et visuelle. Celle-ci en fait également un argot vococentrique, dont le succès auprès des cinéphiles notamment n'est pas une surprise : le slanguage permet de réaliser lexicalement une mise en scène du discours et de reproduire le centrage autour de la voix et du dialogue caractéristique de la narration cinématographique ${ }^{13}$. L'emploi des créations lexicales propres au slanguage peut pourtant dépasser le champ strictement journalistique, et s'ouvrir à celui des professionnels et des spécialistes du cinéma et des médias dans leur ensemble. Leur emploi dans les discours spécialisés peut investir ces créations argotiques d'une valeur terminologique à part entière. Il justifie également à leur sujet l'appellation de néologismes.

\section{Terminologie et néologie}

Le passage que ces créations lexicales peuvent effectuer du lexique potentiel à la frange spécialisée du lexique réel (Tournier 2004:16) indique qu'elles se sont intégrées à un modèle de communication déterminé mais extrinsèque à la revue. Certaines créations propres au slanguage donnent ainsi lieu à une terminologie néologique qui a su s'imposer. Elles peuvent être répertoriées dans des glossaires, dictionnaires et encyclopédies spécialisés ${ }^{14}$. Si nous considérons que la valeur terminologique d'un néologisme se fonde sur sa reconnaissance dans les dictionnaires, entendus comme corpus d'exclusion (Sablayrolles 2008), cette intégration est bien la marque, d'une part, de leur institutionnalisation terminologique. Les termes spécialisés issus du slanguage correspondent, d'autre part, aux deux types de créations néologiques généralement recensées : la néologie de forme et la néologie de sens. Nous observons cependant que 
la néologie propre au slanguage est souvent appelée à dépasser les conventions terminologiques des discours spécialisés.

\subsection{Les mots-valises}

27 La néologie de forme se définit comme l'opération qui « consiste à dénoter une réalité nouvelle [...] et à fabriquer pour ce faire de nouvelles unités lexicales » (Dubois et al. 2001 : 322). Ces nouvelles unités peuvent être créées par composition (Tournier 2004 : 91-98). Or, les nombreuses abréviations du slanguage permettent aisément le télescopage de plusieurs mots, ainsi abrégés, et la création de mots-valises comme biopic (cf. tableau 1). Le mot slanguage lui-même relève à l'évidence de la même opération. Si ces néologismes ne sont pas créés de toutes pièces, leur morphologie néanmoins complexe résulte de l'amalgame d'unités lexicales tronquées (Tournier 2004 : 96-98). Au niveau sémantique, ils suppléent aux carences de la langue là où aucun terme n'existe, par exemple pour désigner l'émergence d'un genre ou d'une forme dramatique. Outre biopic, citons deux autres exemples : dramedy pour dramatic comedy, ou encore sitcom pour situation comedy. Ces mots-valises ont été rapidement intégrés au discours des professionnels et des observateurs des médias, et à la langue courante souvent bien au-delà du monde anglophone. Ces néologismes de forme ne relèvent donc ni d'un discours spécialisé, ni d'un discours de vulgarisation au sens strict. En revanche, l'emploi généralisé de sitcom, par exemple, prouve à quel point la souplesse morphologique et sémantique du slanguage lui permet, en sa qualité même d'argot, de franchir la distance qui sépare plusieurs registres de langue et de s'intégrer facilement à différents types de discours dans des langues diverses.

\subsection{Les mots composés}

Sans qu'ils soient des mots-valises - ils résultent de la seule composition par juxtaposition d'unités lexicales pleinement restituées (Tournier 1987:22) - d'autres mots composés issus du slanguage ont rencontré un grand succès, à l'exemple de soap opera. Il serait difficile de trouver pour cette forme dramatique un terme plus approprié, alors qu'il rapproche à lui seul le pathos quasi opératique des feuilletons télévisés (opera) et la référence commerciale à leurs premiers sponsors liés à l'industrie du savon et de la lessive (soap $)^{15}$. Le succès de ce néologisme se mesure également à l'usage généralisé de sa forme abrégée (soap), une troncation qui témoigne de son emploi dans le discours oral et donc de sa pleine adoption par le grand public. Avec plus ou moins de succès, le slanguage est également à l'origine de mots-valises ou de mots composés comme telefilm, telepic ou cliffhanger.

\subsection{Néologie et fluidité intra- / inter- linguistique}

Qu'elle prenne la forme de mots-valises, de mots composés, voire de mots composés ensuite abrégés, cette néologie s'élabore en conformité avec les structures lexicales de la langue courante. Il s'agit là d'une autre différence qui les dissocie, sur le plan morphologique, des créations lexicales à partir de logos, de formes graphiques, d'onomatopées et d'abréviations. La néologie issue du slanguage constitue donc un exemple représentatif des rapports de distance et de proximité que cet argot entretient, dans son ensemble, avec la langue courante. Il s'en écarte par ses créations 
lexicales impropres, mais un nombre suffisant d'entre elles présente une morphologie assez proche de la langue courante pour qu'elles deviennent des néologismes avérés, et que ceux-ci s'intègrent à leur tour à la langue courante au terme d'un processus intralinguistique nourri d'apports réciproques entre plusieurs types de discours. Ce processus peut même acquérir à la suite une forme de fluidité également interlinguistique, dont les termes franglais sitcom ou soap sont quelques-unes des manifestations.

\subsection{Néologie de forme et de sens}

L'efficacité ludique et séduisante des amalgames, opérés dans les mots-valises, et la clarté morphologique des mots composés, par seule juxtaposition, contribuent à réduire la distance entre la zone sûre du lexique réel et sa frange spécialisée. Ceci explique sûrement, d'une part, la rapidité avec laquelle la terminologie néologique ainsi créée est également adoptée par le grand public. D'autre part, les termes soap opera et cliffhanger introduisent une autre forme de néologie fondée sur sa valeur métaphorique. Elle est remarquable, quant à elle, en raison de sa capacité à frapper les imaginations, au-delà même des barrières d'ordre sociolinguistique et linguistique. Mots composés inédits, ils sont de fait des néologismes de forme. Ils témoignent également d'une néologie de sens qui favorise leur réalisation dans un spectre discursif et linguistique élargi. Ce type de néologie se définit, plus précisément, comme l'opération qui «consiste à employer un signifiant existant déjà dans la langue considérée en lui conférant un contenu qu'il n'avait pas jusqu'alors" (Dubois et al. 2001: 322). Dans le slanguage, ce réemploi de signifiants existants anticipe plus particulièrement la réalisation visuelle de ce qu'ils connotent dans l'imaginaire du lecteur. Nous retrouvons là la forte dimension phatique et visuelle du slanguage; elle confère souvent à ces néologismes la prégnance d'un plan cinématographique dans l'esprit et la mémoire.

\subsection{Néologismes métaphoriques et déterminologisation}

Alors qu'il connote parfaitement la peur du vide et l'irrésolution d'une situation extrême, cliffhanger aura peu de mal à s'imposer comme le néologisme qui désigne la chute d'un épisode de série télévisée. Le message visuel qu'un néologisme comme cliffhanger véhicule s'inscrit donc dans l'esprit du lecteur, ici comme un plan en plongée sur lequel l'image se serait arrêtée, tout autant qu'il s'inscrit dans la trame signifiante du discours. Ceci explique sûrement l'usage généralisé de ces termes, au-delà du discours localisé qui a contribué à leur apparition. Cette généralisation explique toutefois que, pour la plupart d'entre eux, la force du message visuel se soit progressivement estompée pour donner lieu à une métaphore figée, en quelque sorte réduite à un plan fixe, ou à un simple cliché au sens stylistique et photographique du terme. La démétaphorisation du néologisme est alors coextensive d'un phénomène de déterminologisation: le terme d'origine métaphorique devient mot en faisant son entrée dans la langue courante et en perdant, à la suite, sa valeur métaphorique première (Meyer \& Mackintosch 2000). 


\subsection{Une néologie en mouvement}

L'inventivité du slanguage se mesure alors en diachronie à sa créativité néologique et donc à sa capacité de renouvellement terminologique, souvent par métaphores interposées. Elle joue sur une néologie de forme et de sens, particulièrement lorsque la proximité créée entre le lecteur et un néologisme devenu très courant tend à figer la métaphore. La valeur métaphorique du nouveau néologisme crée alors un effet de surprise, devenu nécessaire afin de produire un changement de point de vue. Ce changement permet à la métaphore de retrouver toute sa vivacité. La souplesse sémantique du slanguage lui permet en effet de créer une néologie en mouvement, qui suscite un flux d'images proche de la narration cinématographique. Ainsi l'usure du néologisme métaphorique soap opera donne lieu à la création par suffixation d'un néologisme synonyme, sudser. Celui-ci gagne en intensité visuelle, puisqu'il évoque une forme de plan rapproché sur la mousse de l'eau de lessive (suds). Sont ainsi remétaphorisées l'origine commerciale de ce type de programme et l'effusion de sentiments propre à cette forme dramatique ${ }^{16}$.

\subsection{Une néologie en clin d'œil}

Le néologisme sudser ne fait assurément pas l'objet d'une entrée dans un dictionnaire. Le slanguage reste un argot dont l'inventivité n'a donc pas pour vocation de produire une néologie institutionnalisée dans les discours terminologiques de type métalinguistique. Il est remarquable que ces créations lexicales argotiques s'intègrent dans les discours spécialisés, mais il est peut-être plus remarquable encore qu'un argot soit capable de produire une néologie en mouvement qu'aucune forme de discours ne puisse figer. Le slanguage en tire sa spécificité sociolinguistique et son statut même d'argot, au-delà de toute limite imposée.

Tableau 2. Une néologie en clin d'œil

\begin{tabular}{|l|l|}
\hline Slanguage & Correspondance \\
\hline bird & satellite \\
\hline helmer & film director \\
\hline ozoner & drive-in cinema \\
\hline
\end{tabular}

34 L'évolution presque environnementaliste vers le néologisme ozoner (tableau 2) pour désigner un cinéma en plein air, réduit la distance entre plusieurs domaines de compétences et entre les discours spécialisés qui leur sont associés. Il s'agit bien sûr d'un clin d'œil anodin, mais il marque au passage la proximité que Variety cherche à entretenir avec les nombreuses préoccupations de son très large lectorat, quelles qu'elles soient. Le terme «clin d'œil » pourrait en quelque sorte définir, à lui seul, la plupart de ces néologismes fortement métaphoriques. Ils partagent la vivacité d'un regard amusé qui soulignerait au lecteur un bon mot. La fonction de ces néologismes est donc phatique, et leur potentiel métaphorique confère au discours une dimension 
visuelle, dont l'intensité est surtout calculée pour attirer l'œil et l'attention du lecteur sur un gros titre, ou sur un bon mot au détour d'une phrase.

\subsection{Effet de zoom métonymique}

Cette forme de néologie en clin d'œil peut ainsi résulter d'un déplacement métaphorique, par similitude entre deux éléments: le précipice et un ultime rebondissement, le gouvernail et la direction d'acteurs, un oiseau et un satellite, l'ozone et un cinéma en plein air. Ce déplacement peut aussi être d'origine métonymique, par l'établissement d'un rapport de contiguïté, déjà esquissé dans le terme ozoner.

Tableau 3. Effet de zoom métonymique

\begin{tabular}{|l|l|}
\hline Slanguage & \multicolumn{1}{|c|}{ Correspondance } \\
\hline \hline arthouse & Art cinema \\
\hline \hline hardtop & movie theater \\
\hline to ink & to sign (a contract) \\
\hline to lens & to film \\
\hline to pen & to write (a screenplay) \\
\hline a praisery & a public relations firm \\
\hline
\end{tabular}

Il est plus surprenant encore que le rapport de contiguïté s'établisse uniformément par synecdoque (cf. tableau 3). Il en résulte un rapprochement du tout vers la partie. Comme par un effet de zoom métonymique, ce rapprochement semble alors réduire la distance socioprofessionnelle et socioculturelle qui opposerait le mode de fonctionnement propre à l'organisation verticale d'une industrie (signature de contrats avec un producteur, travail des scénaristes, tournage, campagne de publicité) aux réalités quotidiennes du lecteur de base, alors qu'il ramène chaque étape de cette organisation à des objets ou des produits d'usage courant (de l'encre, un stylo, un objectif ${ }^{17}$. Cet effet de zoom peut également concourir à réduire la distance élitiste qui sépare ordinairement les productions indépendantes et le grand public, parce qu'il associe le cinéma d'art et d'essai (art films) à un centre culturel ouvert à tout public (arthouse). Il est cependant à noter que ces rapprochements ne sont pas nécessairement le fait d'un discours de vulgarisation. Le terme arthouse, par exemple, a été rapidement intégré à la terminologie critique des discours spécialisés et, au-delà, à la langue courante (King 2005 : 13-17).

\section{Néologie et brouillage discursif}

La typologie des règles lexicogéniques que nous venons d'établir témoigne de la grande inventivité du slanguage, tant au niveau morphologique que sémantique. Cependant, 
depuis la lexicalisation de logos, d'onomatopées, d'initiales et autres abréviations jusqu'à la création de néologismes de forme et de sens, chacun de ces procédés concourt prioritairement à une superposition de discours dont résulte un brouillage discursif. C'est de ce brouillage que le slanguage tire sa véritable légitimité éditoriale, puisqu'il confère au discours la capacité d'estomper des divergences d'intérêts (au sens économique, corporatiste et socioculturel). Cette capacité permet à la revue de se démarquer de tout discours formaté, et ainsi de conserver l'adhésion de ses divers lectorats. Nous examinons dans cette dernière partie, la manière dont la néologie propre au slanguage, notamment par suffixation (Tournier 2004:45-50), contribue à estomper, sur le mode péjoratif, les excès d'un discours de type publicitaire ou toute tendance abusive au métadiscours critique.

\subsection{Métaphorisations et suffixations péjoratives}

Un néologisme comme praisery (cf. tableau 3) atteste à la fois d'une néologie de forme, par suffixation, et de sens, du fait qu'il ramène le rôle d'une entreprise en relations publiques à une forme de faire-valoir (to praise), à moins que, par métaphore interposée, il n'évoque aussi une pépinière de talents et de célébrités à partir d'un jeu sur l'homophonie de l'affixe entre praisery et nursery. La pointe de ce néologisme en clin d'œil n'a rien de très appuyée, mais elle n'en demeure pas moins ironique. Praisery constitue donc un exemple de métaphorisation péjorative à l'égard des stratégies de communication, fondées notamment sur la médiatisation propre aux nouvelles formes du star system (Bourget 2005 : 145). Il traduit très bien l'équidistance éditoriale de Variety entre les intérêts des milieux professionnels, soucieux de l'image, et les intérêts du lecteur de base, qui ne doit pas être dupé par ces stratégies. Il en résulte un brouillage discursif qui permet à la revue d'opérer un équilibrage entre des intérêts divergents, et ainsi de maintenir sa ligne éditoriale. Sur le plan morphologique, le néologisme praisery constitue également un bon exemple de la manière dont la néologie dans le slanguage repose sur la suffixation.

\subsection{Le discours publicitaire à l'épreuve du slanguage}

39 Une nouvelle caractéristique du slanguage émerge en effet : toute néologie de forme par suffixation tend à être péjorative. Le néologisme ozoner connote, comme en écho aux enjeux économiques de la distribution des films dans différentes catégories de salles, le détournement des questions écologiques à des fins commerciales dans le discours publicitaire. A flop peut également devenir the year's floppola pour désigner l'échec le plus cuisant de l'année. La suffixation se prête ici à la création d'un néologisme de forme dont la valeur superlative est aberrante. Elle redouble, au niveau morphologique, la particularité, au niveau sémantique, d'une production dont l'échec inattendu s'inscrit comme une aberration dans la logique du discours publicitaire qui annonçait un immense succès commercial. Il en résulte une réduction de l'écart signifiant / signifié, par la proximité redondante créée entre morphème et lexème.

\subsection{Le discours critique à l'épreuve du slanguage}

D'une part, le slanguage peut donc procéder par suffixation dans tout contexte qui se prête à la dérision - l'échec d'un film à gros budget et du discours publicitaire qui en a 
conduit la promotion, par exemple. D'autre part, la suffixation permet au slanguage de pasticher un type de discours critique dans lequel les néologismes suffixés marqueraient une prise de distance au niveau sémantique avec un référent, au point que celui-ci disparaîtrait comme objet du discours au fur et à mesure des suffixations et de l'élargissement incontrôlé du champ dérivationnel (Tournier 2004 : 48-49). Ce type de discours tendrait à une forme de métadiscours, caractérisée par une néologie de forme réduite à quelques suffixes. Le slanguage peut ainsi pasticher cette tendance au métadiscours par la création de termes creux, réduits à un seul suffixe, et signifiant une forme de disproportion: to hypo pour accroitre au-delà de toute commune mesure, ou encore hype pour toute tendance à l'hyperbole, y compris dans le cadre de campagnes promotionnelles.

\subsection{Autonymie et formatage discursif}

Il n'est pas indifférent que ces deux exemples relèvent à la fois du discours critique, qui emprunte souvent à une terminologie d'origine grecque, et du discours de la publicité et des relations publiques. Au niveau sémantique, ce rapprochement entre deux types de discours bien distincts prend pour appui une tendance à l'autonymie dans les deux cas: ressassement théorique d'un côté, et matraquage médiatique de l'autre. L'équidistance éditoriale caractéristique de la revue participe alors à un équilibrage entre dérision et subordination à l'égard de ces deux types de discours. D'une part, Variety est aussi un véhicule publicitaire, notamment lors de la saison des Academy Awards au travers de ses encarts, For Your Consideration, destinés à promouvoir certaines productions auprès des membres du jury et, incidemment, auprès du grand public. D'autre part, la revue est également lue par les spécialistes du cinéma et des médias. Elle peut même contribuer à la constitution d'une terminologie néologique largement répandue. En revanche, le slanguage permet aussi à la revue d'éviter par une néologie en clin d'œil un formatage discursif excessif, qu'il soit fondé sur le discours publicitaire ou sur une tendance au métadiscours critique.

\subsection{Néologie pseudo-générique}

Le traitement du cinéma de genre dans la revue peut, de nouveau, servir d'exemple pour illustrer ce formatage discursif dans les deux cas. Issue d'une stratégie industrielle et commerciale hollywoodienne, telle qu'elle est relayée par le discours formaté des médias, la notion de genre peut scléroser la production cinématographique et le discours critique autour d'un nombre restreint de modèles narratifs et d'enjeux esthétiques, alors que la notion même de genre est hybride et évolutive (Bourget 2005 : 11-12). Or, le slanguage contribue à sa manière à renouveler par dérision la terminologie en la matière. Outre la suffixation, un autre procédé consiste à substituer à un terme générique déjà existant (thriller, film noir, western) une création lexicale dont la portée critique semble être contredite par son origine populaire ou faussement relevée (whodunit, suspenser, horse opera). Cette volonté de renouveler la notion de genre au cinéma peut également passer par la création d'un autre type de néologie pseudogénérique. Celle-ci désigne des genres cinématographiques étrangers, mais elle joue également sur les clichés nationaux de manière politiquement incorrecte. Cette néologie connote ainsi une distance par rapport à un cinéma de genre réduit aux seuls genres américains. Cependant, il n'en résulte qu'une proximité apparente avec ces 
genres cinématographiques étrangers, du fait de la connotation péjorative des néologismes ainsi créés. Par exemple, les chopsockies sont les films d'arts martiaux, par jeu sur la valeur onomatopéique de chop, mais aussi sur le cliché des chopsticks comme emblèmes du cinéma asiatique ${ }^{18}$. La superposition de discours auquel le slanguage participe permet à la revue, ici encore, d'éviter tout positionnement tranché et de se concilier l'adhésion du plus grand nombre de lecteurs, qu'ils soient américains ou étrangers, par un brouillage discursif qui oscille entre humour et ironie, complicité et exclusion.

Dans tous ces exemples, nous avons pu observer la capacité du slanguage à toujours faire varier les indices de distance et de proximité entre plusieurs types de discours, et ainsi à se frayer un chemin entre plusieurs positionnements tranchés, et qui seraient donc intenables au regard de la ligne éditoriale éclectique que la revue s'est tracée. La souplesse morphologique et sémantique de cet argot témoigne en effet d'une grande inventivité phonologique, graphique et métaphorique, qui favorise un brouillage discursif à l'égard de tout discours formaté et contribue ainsi à la souplesse éditoriale de la revue. Grâce au slanguage, Variety s'adapte et peut donner lieu à une variété de discours et s'assurer l'adhésion d'un lectorat le plus large possible. Cette variété justifierait à elle seule le titre de la revue. Elle explique également la diversité des exploitations de Variety auprès des professionnels, du public et des chercheurs. Par fidélité au projet fondateur de Sime Silverman, les colonnes de la revue sont ainsi restées ouvertes à une grande variété de productions, de points de vue et de lectorats.

\section{BIBLIOGRAPHIE}

Altman, R. 1999. Film / Genre. Londres : British Film Institute.

Amiel, V. et P. Coute. 2003. Formes et obsessions du cinéma américain contemporain. Paris :

Klincksieck.

Biskind, P. 2005. Down and Dirty Pictures: Miramax, Sundance and the Rise of Independent Film.

Londres : Bloomsbury Press.

Bourget, J.-L. [1998] 2005. Hollywood : la norme et la marge. Paris : Armand Colin.

Chion, M. 1993. La Voix au cinéma. Coll. Essais. Paris : Cahiers du cinéma.

Dubois, J., M. Giacomo, L. Guespin, C. Marcellesi, J.-B. Marcellesi et J.-P. Mevel. 2001. Dictionnaire

de linguistique et des sciences du langage. Paris : Larousse.

Guiraud, B. 2007. Glossaire bilingue du cinéma et de la vidéo français-anglais / anglais - français. Paris : La Maison du Dictionnaire.

Hayward, S. 1996 (2000). Cinema Studies: The Key Concepts. Londres et New York : Routledge.

Katz, E. [1994] 2001. The Film Encyclopedia. New York : HarperPerennial.

King, G. 2005. American Independent Cinema. Londres et New York : I. B. Tauris 
Lakoff, G. et M. Johnson. 1980. Metaphors We Live By. Chicago et Londres : The University of Chicago Press.

Mercer, J. 1979. Glossary of Film Terms. Houston : University Film Association.

Pearson, R. E. et P. Simpson. 2001. Critical Dictionary of Film and Television Theory. Londres et New York : Routledge.

Meyer, I. et K. Mackintosch 2000. « L'étirement du sens terminologique : aperçu du phénomène de la déterminologisation ». In H. Béjoit et P. Thoiron (dir.). Le Sens en terminologie. Lyon : Presses Universitaires de Lyon, 126-217.

Sablayrolles, J.-F. 2008. « Néologie et dictionnaire(s) comme corpus d'exclusion ». In J.-F. Sablayrolles (dir.). Néologie et terminologie dans les dictionnaires. Paris : Honoré Champion, 19-36.

Schatz, T. (dir.) 2003. Critical Concepts in Media and Cultural Studies. Londres et New York : Routledge.

Scott, J. A. 2005. On Hollywood: the Place, the Industry. Princeton, N.J. :Princeton University Press.

Slide, A. 1998. The New Historical Dictionary of the American Film Industry. Londres et New York: Routledge.

Stoddart, D. 1941. Lord Broadway - Variety's Sime.New York : Wilfred Funk.

Tournier, J. [1987] 2004. Précis de lexicologie anglaise. Paris : Ellipses.

\section{NOTES}

1. Le slanguage est également connu sous le terme de varietyese. La variante headlinese est utilisée pour l'emploi du slanguage dans les manchettes.

2. Voir notamment le numéro spécial, Variety 100, publié en 2005 pour le centenaire de la revue. 03-06-2008 < http://www.variety.com/index.asp?layout=variety100\&content=home>.

3. Voir l'article 'Go west, young pub (Smoke and Mirrors)' dans Variety 100. 03-06-2008 <http:// www.variety.com/article/VR1117938848>.

4. Weekly Variety, Daily Variety Gotham. Gotham est le terme slanguage de New York en référence à Gotham City, la ville du héros de bande dessinée Batman.

5. 03-06-2008 <http://www.variety.com>.

6. 03-06-2008 <http://www.hollywoodreporter.com>.

7. Voir notamment les articles suivants, classés par ordre chronologique de parution 03-06-2008 <http://www.variety.com/article/VR1117976653;htlml?c=2821>;<http://www.variety.com/ article/VR11179801000.html?c=13> ; <http://www.variety.com/article/VR1117983398.htlml? $\mathrm{c}=2513>$.

Voir notamment les deux articles suivants sur la grève des scénaristes de 1988 03-06-2008 <http://www.variety.com/article/VR1117976433.html?c=2821> <http://www.variety.com/ article/VR1117976653?c=2821>.

8. <http://www.variety.com/review/VR1117794786.html?c=93>. Le succès inattendu de Sex, Lies and Videotape, que l'article de Variety a notamment rendu possible, a intéressé de nombreux spécialistes du cinéma contemporain. Alisa Perren montre, par exemple, que ce film marque un véritable renouveau du cinéma indépendant américain dans « Sex, lies and marketing: Miramax and the development of the quality indie blockbuster » (in Schatz 2003, vol. I, 380-97).

9. < http://www.variety.com/index.asp?layout=features2006\&content=jump\&jump=story\&dep>. 
L'ouvrage de Peter Biskind (2005) est une excellente référence sur le rôle joué par le festival de Sundance et la société de production Miramax dans l'essor du cinéma indépendant depuis la fin des années 80 .

10. 03-06-2008 <http://www.variety.com/article/VR1117922332.html?c=21>.

11. La traduction même du mot slanguage nous permet de mesurer très rapidement la souplesse morphologique de cet argot. Suggestions de traduction par équivalence : argocode, esc-argot.

12. Les occurrences qui illustrent cette typologie sont définies dans le dictionnaire du slanguage accessible sur le site de Variety 03-06-2008 < http://www.variety.com/index.asp?/ layout=slanguage>.

13. Le vococentrisme est un concept critique que j'emprunte aux études cinématographiques. Il désigne le processus par lequel, dans un ensemble sonore, la voix attire et centre notre attention, de la même façon que l'œil, dans un plan de cinéma, est attiré par le visage humain (Chion 1993 : 18-20).

14. Voir Guiraud (2007), Hayward [1996] 2000, Katz [1994] 2001, Mercer 1979, Pearson \& Simpson 2001 et Slide 1998. Ont été sélectionnés les ouvrages qui présentent le plus d'entrées axées sur le vocabulaire technique, économique et esthétique du cinéma et de la télévision.

15. Le premier soap opera, intitulé As The World Turns, est lancé aux États-Unis en 1956. Variety a consacré un article exclusif, publié pour le cinquantième anniversaire de cette série, à l'émergence et au succès à très long terme de ce format télévisuel (06-08-2008 <http:// www.variety.com/article/VR1117940666.html>).

16. La concurrence entre Variety et d'autres publications peut également être à l'origine de la création de sudser. Ceci confirmerait qu'une exclusivité journalistique peut reposer, en totalité ou en partie, sur l'accroche constituée par une création lexicale (cf. 3.6). En effet, que le terme soap opera soit directement issu du slanguage est sujet à caution. Si Variety a consacré environ 3000 articles à ce format télévisuel depuis qu'il a été lancé, le terme n'apparaît que rarement en gros titre. Time Magazine semblerait ainsi avoir ravi à la revue spécialisée l'exclusivité de ce terme en titrant, en couverture de son numéro du 12 janvier 1976, Soap Operas: Sex and Suffering in the Afternoon. Dans ces conditions, la création d'un terme synonyme exclusif comme sudser se serait imposée à la rédaction de Variety avec d'autant plus de force.

17. Des cognitivistes comme Lakoff et Johnson considèrent que la métaphore et notamment la métonymie sont les procédés les plus courants pour se référer aux individus, objets et situations dans la vie quotidienne : "Metonymic concepts (like the part for the whole) are part of the ordinary way we think and act as well as talk. » (1980:39).

18. John G. Calweti dresse un bilan exhaustif sur l'intégration par l'industrie hollywoodienne des genres cinématographiques venant d'Asie dans "Chinatown and generic transformation in recent American films » (in Schatz 2003, vol. I, 80-95).

\section{RÉSUMÉS}

Variety se définit comme une publication corporatiste, spécialisée et grand public. L'article se concentre sur la manière dont le slanguage, l'argot journalistique utilisé dans la revue, contribue au niveau du discours à l'établissement d'une ligne éditoriale éclectique, destinée à consolider l'adhésion d'un vaste lectorat. Cet éclectisme est tout d'abord mis à l'épreuve du positionnement de Variety face aux intérêts respectifs de l'industrie hollywoodienne et du secteur indépendant. 
La fonction éditoriale du slanguage est alors resituée dans l'histoire de la revue telle qu'elle reflète les évolutions de l'industrie du divertissement, leurs enjeux économiques et artistiques, et la création concomitante d'une terminologie appropriée. L'article inclut une typologie de règles lexicogéniques qui contribuent à la formation de néologismes dont l'emploi dans des contextes variés estompe les limites préétablies entre plusieurs types de discours. Cet effet de brouillage discursif, renforcé par de fréquents déplacements métaphoriques, confère au slanguage une forte dimension phatique et visuelle. Il assure un équilibrage éditorial entre le matraquage publicitaire et les excès du métadiscours critique.

Variety defines itself as a trade paper with a high circulation. The article focuses on how slanguage, the form of journalese used in Variety, helps at the discursive level to establish an eclectic editorial line, aimed at comforting a wide readership. This eclecticism is first tested against the position of the magazine regarding the specific interests of the Hollywood film industry and the independent sector. The editorial function of slanguage is then set within the history of Variety as reflecting the many developments of the entertainment industry, their economic and artistic relevance, and the attendant creation of an appropriate terminology. The article includes a typology of lexicogenic rules that contribute to the formation of neologisms used in a wide range of contexts, thus blurring the borderline between several types of discourses. That discursive blur effect, reinforced by frequent metaphoric shifts, gives to slanguage a strong phatic and visual quality. It guarantees a form of editorial equilibrium between promotional hype and the excesses of critical metadiscourse.

\section{INDEX}

Mots-clés : cinéma, discours, métadiscours, métaphorisation, néologie, terminologie

Keywords : cinema, discourse, metadiscourse, metaphorisation, neology, terminology

\section{AUTEUR}

\section{FRANÇOIS ROPERT}

François Ropert, docteur ès lettres, est agrégé d'anglais à l'Université Paris 1 Panthéon-Sorbonne. Ses recherches portent sur l'anglais de spécialité (secteur sciences humaines), la littérature et le cinéma. Il enseigne en Licence et Master d'histoire et d'esthétique du cinéma à Paris 1 et en Licence de cinéma à Paris 3 Sorbonne Nouvelle. Il est également chargé d'enseignement pour le CNED (secteurs arts plastiques et cinéma). fran.ropert@wanadoo.fr 